\title{
FIBRATIONS AND CONTACT STRUCTURES
}

\author{
HAMIDOU DATHE AND PHILIPPE RUKIMBIRA
}

Received 18 May 2004 and in revised form 8 October 2004

We prove that a closed 3-dimensional manifold is a torus bundle over the circle if and only if it carries a closed nonsingular 1 -form which is linearly deformable into contact forms.

\section{Introduction}

A contact form on a $(2 n+1)$-dimensional manifold $M$ is a 1 -form $\alpha$ such that $\alpha \wedge(d \alpha)^{n}$ is a volume form on $M$. The system of equations $\alpha(Z)=1$ and $d \alpha(Z, X)=0$ for arbitrary $X$ uniquely determines a vector field $Z$ called the Reeb vector field or the characteristic vector field of $\alpha$. The tangent subbundle $\xi=\operatorname{kern} \alpha$ of rank $2 n$ is called the contact structure associated with $\alpha$. In general, a contact structure on a $(2 n+1)$-dimensional manifold is a rank $2 n$ tangent subbundle which is locally determined by contact forms (see Blair's book [1] for more details about contact structures).

The manifolds in this paper will be assumed to be oriented and all the plane fields considered herein are supposed to be transversely orientable. Let $\xi$ be a hyperplane field on a manifold $M$. When $\xi$ is a foliation, we say that $\xi$ is deformable into contact structures if there exists a one parameter family $\xi_{t}$ of hyperplane fields satisfying $\xi_{0}=\xi$ and for all $t>0, \xi_{t}$ is contact. It is well known from Eliashberg and Thurston's work [3, page 31, Theorem 2.4.1] that any oriented codimension $1, C^{2}$-foliation on an oriented 3-manifold can be perturbed into contact structures, except the product foliation of $\mathbf{S}^{2} \times \mathbf{S}^{1}$ by spheres $\mathbf{S}^{2}$. It was then unknown if this approximation can always be done through a deformation. In this paper, we deal with particular deformations called "linear." For a foliation $\xi$ defined by a 1 -form $\alpha_{0}$, a deformation $\xi_{t}$ defined by 1 -forms $\alpha_{t}$ is said to be linear if $\alpha_{t}=\alpha_{0}+t \alpha$ where $\alpha$ is a 1 -form on $M$ (independent of $t$ ). We point out that our definition of linearity is weaker than that of Eliashberg and Thurston [3, page 23]. In [2], the following theorem was proved.

Theorem 1.1. Let $M$ be a closed, $(2 n+1)$-dimensional manifold, $\alpha_{0}$ a closed 1 -form on $M$, and $\alpha$ any 1 -form on $M$. Then, the following two conditions are equivalent.

(i) The 1 -forms $\alpha_{t}=\alpha_{0}+$ t $\alpha$ in a linear deformation of $\alpha_{0}$ are contact for all $t>0$.

(ii) The 1 -form $\alpha$ is contact and $\alpha_{0}(Z)=0$ where $Z$ is the Reeb vector field of $\alpha$. 
Using this theorem, we will obtain a characterization of $\mathbf{T}^{2}$-bundles over $\mathbf{S}^{1}$.

\section{Torus bundles over $S^{1}$}

In dimension 3, one has the following characterization of $\mathbf{T}^{2}$-bundles over $\mathbf{S}^{1}$.

Theorem 2.1. Let $M$ be a closed, 3-dimensional manifold. Then the following two conditions are equivalent.

(i) There is on $M$ a closed nonsingular 1-form $\alpha_{0}$ and a 1-form $\alpha$ such that the 1-forms $\alpha_{t}=\alpha_{0}+t \alpha$ in a linear deformation of $\alpha_{0}$ are contact for all $t>0$.

(ii) $M$ is a $\mathbf{T}^{2}$-bundle over $\mathbf{S}^{1}$.

Proof. First we prove that (i) implies (ii). If $\alpha_{0}$ and $\alpha$ are as in (i), then, by Theorem 1.1, $\alpha$ is a contact form whose characteristic vector field $Z$ satisfies $\alpha_{0}(Z)=0$. Arbitrarily close to $\alpha_{0}$, there is a closed, rational 1 -form $\beta$ which is obtained as follows. By the Hodge-de Rham theorem, $\alpha_{0}=h+d f$ where $h$ is a harmonic 1 -form and $f$ is a smooth function. Let $h_{1}, \ldots, h_{l}$ be a basis of the vector space of harmonic forms on $M$. Then $h=\lambda_{1} h_{1}+\cdots+$ $\lambda_{l} h_{l}$ where the numbers $\lambda_{i}$ 's represent the periods of $h$. One can arbitrarily approximate each of $\lambda_{i}$ by a rational number $q_{i}$ and consider the 1 -form $\beta=q_{1} h_{1}+\cdots+q_{l} h_{l}+d f$. If the approximations $q_{i}$ are close enough, then $\beta$ is a nonsingular, closed 1-form with rational periods. The leaves of the foliation $\operatorname{kern} \beta$ form a fibration of $M$ by closed surfaces $\Sigma$ over $\mathbf{S}^{1}$. Let $g$ be any metric on $M$ in which $\beta$ is of unit length and let $N$ denote the metric dual unit vector field of $\beta$. Let $\operatorname{Pr}(Z)=Z-\beta(Z) N$ be the orthogonal projection of $Z$ onto $\operatorname{kern} \beta$. Suppose that at some point $x \in M, \operatorname{Pr}(Z)=0$. Then, at that point, $Z=$ $\beta(Z) N$ and therefore $0=\alpha_{0}(Z)=\beta(Z) \alpha_{0}(N)$. If $\beta$ and $\alpha_{0}$ are sufficiently close, then $\alpha_{0}(N)$ is close to $\beta(N)=1$ and hence $\alpha_{0}(N) \neq 0$, which implies that $\beta(Z)=0$. So, $\operatorname{Pr}(Z)=0$ at some point implies that $Z=0$ at that particular point, contradicting the fact that $Z$ is a nonsingular vector field. Therefore, $\operatorname{Pr}(Z)=Z-\beta(Z) N$ is a nonsingular vector field. Moreover, $\beta(\operatorname{Pr}(Z))=0$, hence $\operatorname{Pr}(Z)$ is everywhere tangent to the fiber $\Sigma$, which means that $\Sigma$ is a 2 -torus $\mathbf{T}^{2}$.

To prove that (ii) implies (i), we will exhibit on every $\mathbf{T}^{2}$-bundle over $\mathbf{S}^{1}$ a contact form $\alpha$ whose characteristic vector field $Z$ is tangent to the fibers $\mathbf{T}^{2} . \alpha_{0}$ can then be taken to be the pullback of $d \theta$, the volume form on $\mathbf{S}^{1}$, under the fiber projection map. Suppose that $M$ is an orientable $\mathbf{T}^{2}$-bundle over $\mathbf{S}^{1}$ with monodromy $A \in \mathrm{SL}_{2} \mathbb{Z}$. Following Geiges and Gonzalo [5], we consider 3 different cases.

Case 1 ( $\operatorname{trace} A \geq 3$ ). The manifold $M$ is a left quotient of $\mathrm{Sol}^{3}$, the solvable Lie group defined as a split extension of $\mathbb{R}^{2}$ by $\mathbb{R}$. The group $\mathbb{R}$ acts on $\mathbb{R}^{2}$ by $z \cdot(x, y)=\left(e^{z} x, e^{-z} y\right)$. Sol ${ }^{3}$ can be identified with $\mathbb{R}^{3}$ with the following multiplication:

$$
\left(x_{0}, y_{0}, z_{0}\right) \cdot(x, y, z)=\left(x_{0}+e^{z_{0}} x, y_{0}+e^{-z_{0}} y, z_{0}+z\right) \text {. }
$$

The bundle projection is given by $(x, y, z) \rightarrow z$ (see [7]).

On $\mathrm{Sol}^{3}$, set

$$
\alpha=\cos \left(\frac{2 \pi n}{\gamma} z\right) e^{-g(z)} d x-\sin \left(\frac{2 \pi n}{\gamma} z\right) e^{g(z)} d y
$$


$n \in \mathbb{N}, g$ is a smooth, monotone increasing function that satisfies

(i) $g(z)=z$ near $z=0$,

(ii) $g(z)=\gamma / 2$ near $z=\gamma / 2$,

(iii) $g(z+\gamma)=g(z)+\gamma$ for all $z$.

The 2 -form $d \alpha$ is given by

$$
\begin{aligned}
d \alpha= & -\left[\frac{2 \pi n}{\gamma} \sin \left(\frac{2 \pi n}{\gamma} z\right) e^{-g(z)}+g^{\prime}(z) e^{-g(z)} \cos \left(\frac{2 \pi n}{\gamma} z\right)\right] d z \wedge d x \\
- & {\left[\frac{2 \pi n}{\gamma} \cos \left(\frac{2 \pi n}{\gamma} z\right) e^{g(z)}+g^{\prime}(z) e^{g(z)} \sin \left(\frac{2 \pi n}{\gamma} z\right)\right] d z \wedge d y, } \\
\alpha \wedge d \alpha= & {\left[\frac{2 \pi n}{\gamma}+g^{\prime}(z) \cos \left(\frac{2 \pi n}{\gamma} z\right) \sin \left(\frac{2 \pi n}{\gamma} z\right)\right] d x \wedge d y \wedge d z . }
\end{aligned}
$$

Since the coefficient in $\alpha \wedge d \alpha$ is bigger than $2 \pi n / \gamma-g^{\prime}(z)$, one sees that for $n$ large so that $2 n \pi / \gamma-g^{\prime}(z)>0, \alpha$ defines a contact form on $\mathrm{Sol}^{3}$ that is invariant under $\Gamma$, the discrete subgroup generated by elements of the form $\left(\alpha_{1}, \beta_{1}, 0\right),\left(\alpha_{2}, \beta_{2}, 0\right),(0,0, \gamma)$. Moreover, the characteristic vector field $Z$ of the induced contact form is given by

$$
F(x, y, z) \partial x+G(x, y, z) \partial y
$$

for some functions $F$ and $G$ on $M$. Therefore, $Z$ is tangent to the fibers of the projection map Sol ${ }^{3} \rightarrow \Gamma \backslash \mathrm{Sol}^{3}$.

Case 2 (trace $A<-3$ ). The manifold $M$ is diffeomorphic to a quotient of the form $\Gamma \backslash \mathrm{Sol}^{3}$ where $\Gamma$ is generated by elements $\left(\alpha_{1}, \beta_{1}, 0\right),\left(\alpha_{2}, \beta_{2}, 0\right)$ in $\mathrm{Sol}^{3}$ and a generator (see [4])

$$
(x, y, z) \longrightarrow\left(-e^{\gamma} x,-e^{-\gamma} y, z+\gamma\right) .
$$

Set

$$
\alpha=\cos \left(\frac{n \pi z}{\gamma}\right) e^{-g(z)} d x-\sin \left(\frac{n \pi z}{\gamma}\right) e^{g(z)} d y
$$

for odd $n$. The same calculations as in the previous case show that $\alpha$ induces a contact form on $\Gamma \backslash \mathrm{Sol}^{3}$ whose characteristic vector field is tangent to the fibers of the projection map.

Case 3 (the trace of the monodromy matrix $A$ satisfies $|\operatorname{trace} A| \leq 2$ ). In this case (see [6]), either $A$ is a periodic matrix or it is conjugate to $\pm A_{k}$,

$$
A_{k}=\left(\begin{array}{ll}
1 & k \\
0 & 1
\end{array}\right) \text {, }
$$

$k \in \mathbb{Z} /\{0\}$. 
Subcase 1 ( $A$ is conjugate to $A_{k}$ ). The manifold $M$ is a left quotient of $\mathrm{Nil}^{3}$. $\mathrm{Nil}^{3}$ can be thought of as $\mathbb{R}^{3}$ with the multiplication

$$
\left(x_{0}, y_{0}, z_{0}\right) \cdot(z, y, z)=\left(x_{0}+x, y_{0}+y, z_{0}+z+x_{0} y\right) \text {. }
$$

Every compact left quotient of $\mathrm{Nil}^{3}$ is diffeomorphic to one of the form $\Gamma_{k} \backslash \mathrm{Nil}^{3}, k \in$ $\mathbb{Z} /\{0\}$, where $\Gamma_{k}$ is generated by elements $(k, 0,0),(0,1,0),(0,0,1)$. The left quotients are precisely the $\mathbf{T}^{2}$-bundles over $\mathbf{S}^{1}$ with monodromy $A_{k}$, the bundle projection being given by $(x, y, z) \rightarrow y$.

Set

$$
\alpha=\cos (2 \pi y) d x-\sin (2 \pi y)(d z-f(x) d y),
$$

where $f$ is a smooth, monotone increasing function that satisfies

(i) $f(x)=x$ near $x=0$,

(ii) $f(x)=k / 2$ near $x=k / 2$,

(iii) $f(x+k)=f(x)+k$ for all $x$.

One sees that $d \alpha$ is given by

$$
\begin{gathered}
d \alpha=-2 \pi \sin (2 \pi y) d y \wedge d x-2 \pi \cos (2 \pi y) d y \wedge d z+f^{\prime}(x) \sin (2 \pi y) d x \wedge d y, \\
\alpha \wedge d \alpha=-\left[2 \pi+f^{\prime}(x) \sin ^{2}(2 \pi y)\right] d x \wedge d y \wedge d z .
\end{gathered}
$$

Since $2 \pi+f^{\prime}(x) \sin ^{2}(2 \pi y)$ is strictly positive, the 1 -form $\alpha$ induces a contact form on $\Gamma_{k} \backslash \mathrm{Nil}^{3}$ whose characteristic vector field $Z$ is given by

$$
F(x, y, z) \partial x+G(x, y, z) \partial z
$$

for some functions $F$ and $G$ on $M . Z$ is therefore tangent to the fibers of the $\mathrm{T}^{2}$ fibration. Subcase 2 ( $\mathbf{T}^{2}$-bundles over $\mathbf{S}^{1}$ with periodic monodromy). These are compact left quotients of $\widetilde{\mathbf{E}}_{2}$, the universal cover of the group of Euclidean motions in the plane. The Lie algebra of $\widetilde{\mathbf{E}}_{2}$ admits a basis $\left\{e_{1}, e_{2}, e_{3}\right\}$ with brackets $\left[e_{1}, e_{2}\right]=0,\left[e_{3}, e_{1}\right]=e_{2}$, $\left[e_{2}, e_{3}\right]=e_{1}$.

Let $\omega_{1}, \omega_{2}, \omega_{3}$ be the dual coframe of left invariant 1 -forms on $\widetilde{\mathbf{E}}_{2} . \omega_{1}$ and $\omega_{2}$ are contact forms, $\omega_{3}$ is a closed 1-form. The foliation defined by $\omega_{3}$ passes down to any left quotient of $\widetilde{\mathbf{E}}_{2}$ into the fibration by $\mathbf{T}^{2}$. Each of the contact forms $\omega_{1}, \omega_{2}$ passes down to the $\mathbf{T}^{2}$ bundle, with characteristic vector field tangent to the fibers $\mathbf{T}^{2}$.

On a $\mathbf{T}^{2}$-bundle over $\mathbf{S}^{1}$, it is not just the pullback of the volume form on $\mathbf{S}^{1}$ that is linearly deformable into contact forms. One has the following proposition.

Proposition 2.2. Let $M$ be a $\mathbf{T}^{2}$-bundle over $\mathbf{S}^{1}$. Then any rational, nonsingular, closed 1 -form on $M$ is linearly deformable into contact forms.

Proof. Let $\alpha_{0}$ be a rational, nonsingular, closed 1-form on $M . \alpha_{0}$ defines a $\Sigma$-fibration of $M$ over $S^{1}$, where $\Sigma$ is a closed surface. Since the homotopy groups $\pi_{p}\left(\mathbf{S}^{1}\right)$ are trivial for $p \geq 2$, the homotopy exact sequences of the two fibrations contain the following short 
exact sequences:

$$
\begin{aligned}
& 0 \longrightarrow \pi_{1}(\Sigma) \longrightarrow \pi_{1}(M) \longrightarrow \mathbb{Z} \longrightarrow 0 \\
& 0 \longrightarrow \pi_{1}\left(\mathbf{T}^{2}\right) \longrightarrow \pi_{1}(M) \longrightarrow \mathbb{Z} \longrightarrow 0 .
\end{aligned}
$$

It follows that $\Sigma$ has to be a torus. The rest of the proof is as the proof of (ii) implies (i) from Theorem 2.1.

Corollary 2.3. Any fibration over the circle $\mathbf{S}^{1}$ on the three-dimensional torus $\mathbf{T}^{3}$ is linearly deformable into contact structures.

In [2], we give an example of transversely affine foliation which is linearly deformable into contact structures. Here, we will prove the following generalization of this example.

Proposition 2.4. On a three dimensional closed manifold, every transversely affine foliation of codimension 1 with holonomy is linearly deformable into contact structures.

Proof. The proof is based on the principe of deforming a foliation near a curve of nontrivial holonomy (see [3]). It is known that (see [8]) a transversely affine foliation with nontrivial holonomy on a compact manifold has only finitely many compact leaves and each of them has nontrivial holonomy, hence a curve with nontrivial holonomy.

A natural question is the following: can one generalize Theorem 1.1 to all transversely affine foliations? The answer is no. Here is a counterexample.

Let $M$ be a $\mathrm{T}^{2}$-bundle over the circle with monodromy $A$ satisfying $\operatorname{Tr} A>2$. $M$ can be identified with some left quotient of $\mathrm{Sol}^{3}$ (see notations in the proof of Theorem 2.1, Case 1). Let $\lambda$ be the eigenvalue of $A$ such that $\lambda>1, u$ and $v$ the eigenvectors corresponding to $\lambda$ and $1 / \lambda$.

On $\mathrm{Sol}^{3}$, set the vector fields $X=-1 /(\log \lambda) \partial /(\partial z), Y=\lambda^{-z} u$, and $Z=\lambda^{z} v$. These vector fields induce three vector fields on $M$ also denoted by $X, Y, Z$ and which satisfy the identities $[X, Y]=Y,[X, Z]=-Z,[Y, Z]=0$. The duals of these vector fields are nonsingular 1-forms $\omega_{1}, \omega_{2}$, and $\omega_{3}$ satisfying $d \omega_{1}=0, d \omega_{2}=\omega_{1} \wedge \omega_{2}$, and $d \omega_{3}=-\omega_{1} \wedge$ $\omega_{3}$. Let $\mathscr{F}$ denote the codimension 1 foliation defined by kern $\omega_{2}$. $\mathscr{F}$ is a transversely affine foliation on $M$. For all $t \geq 0$, the 1 -forms $\alpha_{t}=\omega_{2}+t \omega_{3}$ define a linear deformation of $\mathscr{F}$ into contact structures; but the 1 -form $\omega_{3}$ is not contact.

\section{References}

[1] D. E. Blair, Riemannian Geometry of Contact and Symplectic Manifolds, Progress in Mathematics, vol. 203, Birkhäuser Boston, Massachusetts, 2002.

[2] H. Dathe and P. Rukimbira, Foliations and contact structures, Advances in Geometry 4 (2004), no. $1,75-81$.

[3] Y. M. Eliashberg and W. P. Thurston, Confoliations, University Lecture Series, vol. 13, American Mathematical Society, Rhode Island, 1998.

[4] H. Geiges, Symplectic structures on $T^{2}$-bundles over $T^{2}$, Duke Math. J. 67 (1992), no. 3, 539555.

[5] H. Geiges and J. Gonzalo, Contact circles on 3-manifolds, J. Differential Geom. 46 (1997), no. 2, 236-286. 
[6] K. Sakamoto and S. Fukuhara, Classification of $T^{2}$-bundles over $T^{2}$, Tokyo J. Math. 6 (1983), no. 2, 311-327.

[7] P. Scott, The geometries of 3-manifolds, Bull. London Math. Soc. 15 (1983), no. 5, 401-487.

[8] B. Seke, Sur les structures transversalement affines des feuilletages de codimension un, Ann. Inst. Fourier (Grenoble) 30 (1980), no. 1, 1-29 (French).

Hamidou Dathe: Département de Mathématique, Physique et Informatique, Faculté des Sciences et Techniques, Université Cheikh Anta Diop (UCAD), Dakar, Senegal

E-mail address: hdathe@ucad.sn

Philippe Rukimbira: Department of Mathematics, Florida International University, Miami, FL 33199, USA

E-mail address: rukim@fiu.edu 


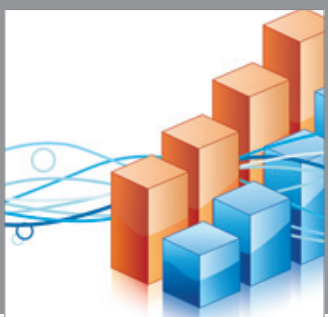

Advances in

Operations Research

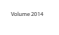

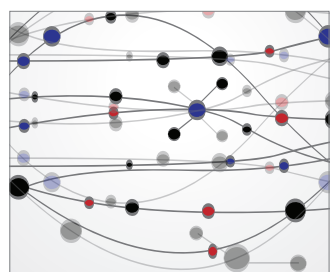

\section{The Scientific} World Journal
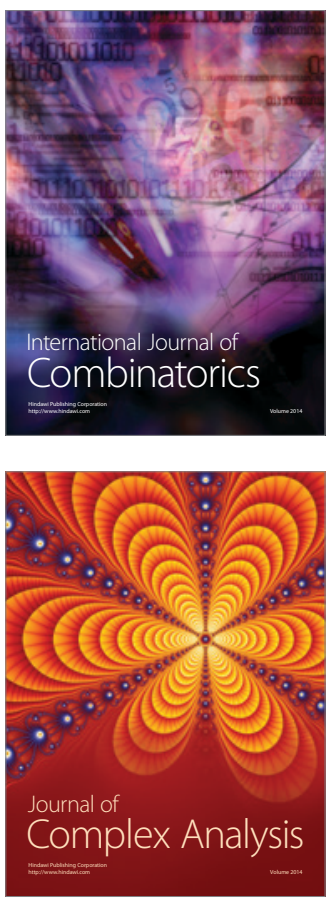

International Journal of

Mathematics and

Mathematical

Sciences
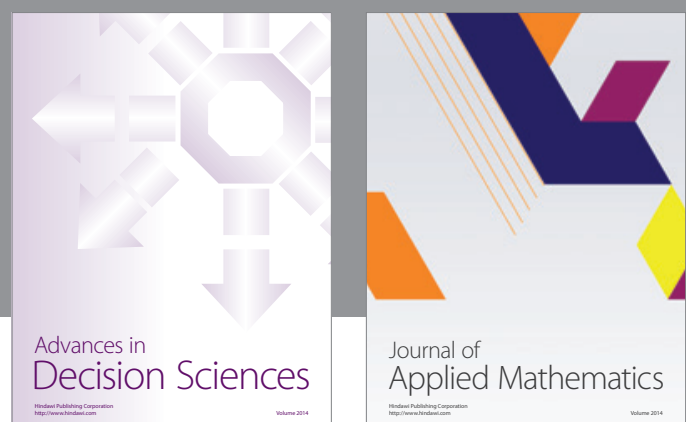

Journal of

Applied Mathematics
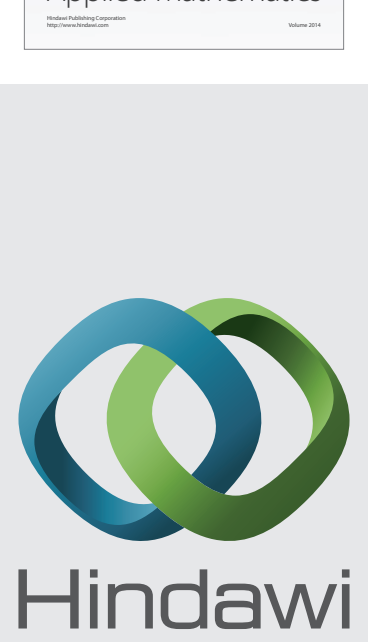

Submit your manuscripts at http://www.hindawi.com
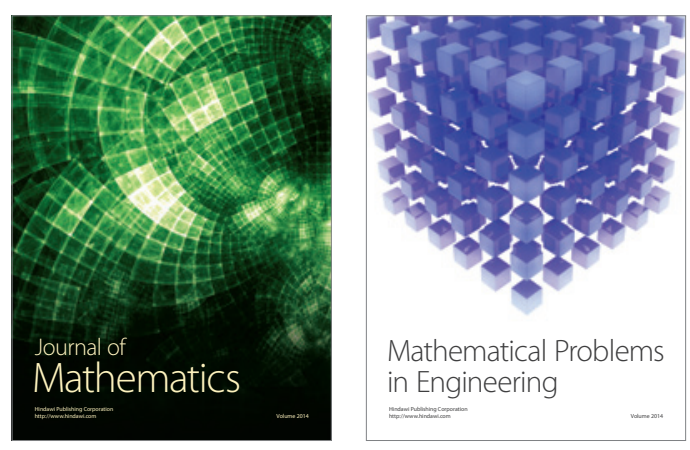

Mathematical Problems in Engineering
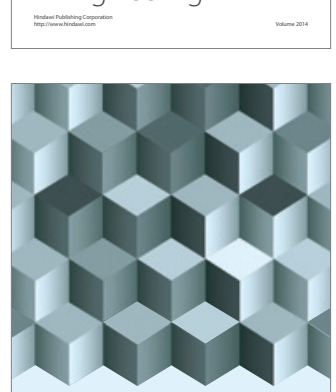

Journal of

Function Spaces
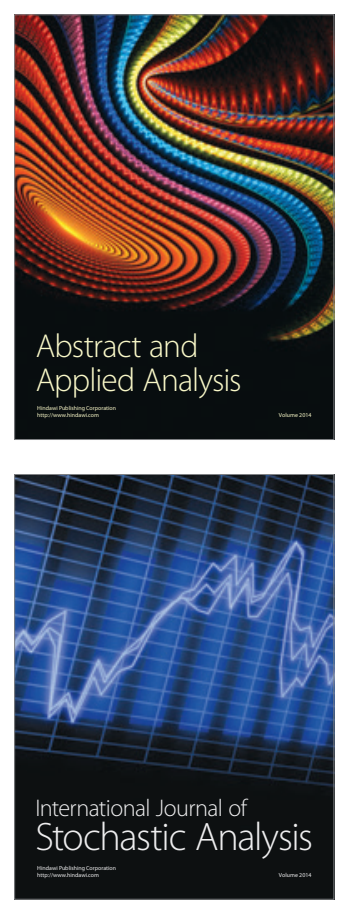

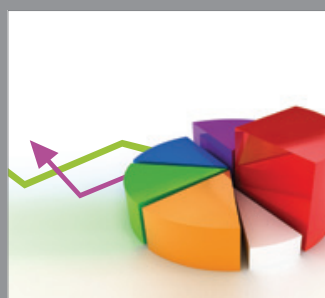

ournal of

Probability and Statistics

Promensencen
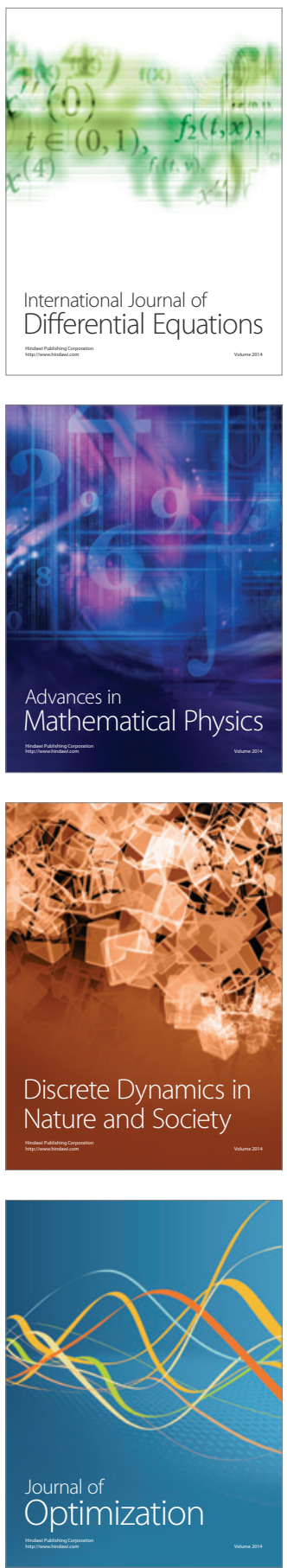